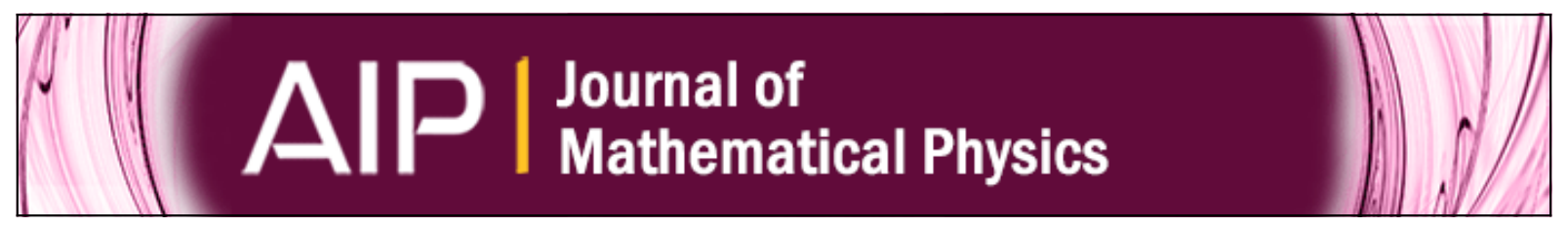

\title{
A discrete-velocity, stationary Wigner equation
}

Anton Arnold, Horst Lange, and Paul F. Zweifel

Citation: Journal of Mathematical Physics 41, 7167 (2000); doi: 10.1063/1.1318732

View online: http://dx.doi.org/10.1063/1.1318732

View Table of Contents: http://scitation.aip.org/content/aip/journal/jmp/41/11?ver=pdfcov

Published by the AIP Publishing

\section{AlP Re-register for Table of Content Alerts}




\section{A discrete-velocity, stationary Wigner equation}

Anton Arnold ${ }^{\text {a) }}$

Fakultät für Mathematik und Informatik, Universität des Saarlandes,

Postfach 1511 50, D-66041 Saarbrücken, Germany

Horst Lange ${ }^{\text {b) }}$

Mathematisches Institut, Universität Köln, Weyertal 86-90, D-50931 Köln, Germany

Paul F. Zweifel ${ }^{\mathrm{c})}$

Physics Department, Virginia Tech, Blacksburg, Virginia 24061-0435

(Received 27 April 1999; accepted for publication 22 August 2000)

This paper is concerned with the one-dimensional stationary linear Wigner equation, a kinetic formulation of quantum mechanics. Specifically, we analyze the well-posedness of the boundary value problem on a slab of the phase space with given inflow data for a discrete-velocity model. We find that the problem is uniquely solvable if zero is not a discrete velocity. Otherwise one obtains a differential-algebraic equation of index 2 and, hence, the inflow data make the system overdetermined. (C) 2000 American Institute of Physics.

[S0022-2488(00)00112-2]

\section{INTRODUCTION}

The so-called Wigner distribution function was introduced as a method for reformulating quantum mechanics in classical phase space ${ }^{1}\left(\mathbb{R}^{6 N}\right.$ for a general $N$-particle system; however we consider here only systems which are one dimensional and described by a mean, or self-consistent, field, so our phase space is $\mathbb{R}^{2}$ ).

The (real-valued) Wigner function, $w(x, v, t)$ with $x \in \mathbb{R}, v \in \mathbb{R}$, and $t \in \mathbb{R}^{+}$is a quasiprobability distribution; it is not, in general, positive but its marginal distribution, the configuration-space density

$$
n(x, t)=\int_{\mathbb{R}} w(x, v, t) d v
$$

is indeed non-negative and, in fact, corresponds exactly with the correct quantum-mechanical expression for the density. For an arbitrary quasiprobability distribution $w(x, v)$ to be a (physical) Wigner function, it has to correspond to a positive trace class operator, the density matrix (cf. Ref. 2).

Wigner showed in Ref. 1 that $w$ obeys a kinetic (quasitransport) equation, the so-called Wigner equation

$$
w_{t}+v w_{x}-\Theta[V] w=0,
$$

with the pseudodifferential operator

$$
\Theta[V]=i\left[V\left(x+\frac{\partial_{v}}{2 i}\right)-V\left(x-\frac{\partial_{v}}{2 i}\right)\right],
$$

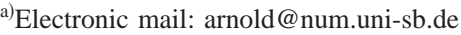

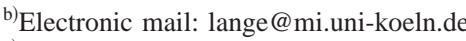

${ }^{c}$ Electronic mail: zweifel@vt.edu
} 
where we have set Planck's constant $\hbar=1$. Alternatively, $\Theta[V]$ can (formally) be expressed as a convolution operator in $v$ :

$$
(\Theta[V] w)(x, v)=a(x, v) *{ }_{v} w(x, v),
$$

with

$$
a(x, v)=\sqrt{\frac{8}{\pi}} \operatorname{Im}\left[e^{2 i v x}(\mathcal{F} V)(2 v)\right],
$$

and $\mathcal{F}$ denotes the Fourier transform. These two definitions of $\Theta[V]$ coincide under some regularity and decay assumptions on $V$ (cf. Ref. 3).

In (1.2b) $V$ represents the (real-valued) system potential. It may either be specified ab initio (the linear case) or may be a self-consistent potential which depends on the density $n$ [Eq. (1.1)] (the nonlinear case). In a typical situation $V$ obeys a Poisson equation and one speaks of the Wigner-Poisson system. However, in this paper only the linear stationary Wigner equation is studied. Furthermore, as suggested by the title of the paper, we are interested in the stationary equation.

In the last several decades many physicists have used the Wigner formalism for quantum scattering theory $;{ }^{4,5}$ for computing virial coefficients; ${ }^{4}$ " squeezed states;", ${ }^{6}$ and for Hartree-Fock calculations. ${ }^{7}$ When considered on the whole space, i.e., $x \in \mathbb{R}$, the Wigner approach is equivalent to "ordinary" quantum mechanics. On finite (spatial) domains, however, it is tricky for three reasons: First, the potential appearing in $\Theta[V]$ must still be known in the whole space, here $\mathbb{R}_{x}$. Second, it is not clear how to formulate adequate boundary conditions (BCs). ${ }^{8}$ Third, it is usually unknown if the Wigner function $w(x, v)$ on a bounded domain corresponds to a positive density matrix operator that gives rise to a non-negative density $n(x)$.

For the past 15 years physicists, engineers, and mathematicians have been using Wigner equation models to simulate the electron transport in submicron semiconductor devices (see, e.g., Refs. 9 and 10). In these numerical simulations physicists, ${ }^{9,11}$ have mostly used so-called inflow boundary conditions for the Wigner equation. These classical transport-theoretical BCs give reasonable results even for quantum models, if they are applied "far enough away" from the main source of quantum effects (e.g., tunneling barriers). Assuming the boundaries are in the onedimensional model at $x=0$ and $x=L$ one specifies the distribution flowing into a medium through the boundary (either in the stationary or the time-dependent case):

$$
\begin{gathered}
w(0, v)=f^{+}(v), \quad v>0, \\
w(L, v)=f^{-}(v), \quad v<0 .
\end{gathered}
$$

In Refs. 12 and 13 absorbing boundary conditions have been devised for the Wigner equation as a refinement of inflow BCs. They account for the coupling of the incoming and outgoing distribution at the boundary in quantum kinetic models.

We remark that both of these BCs break the strict correspondence between the Wigner and Schrödinger-Heisenberg formulations of quantum mechanics. Hence, it is not easy to judge if the resulting Wigner function is physical in the sense of corresponding to a positive density matrix. It would, for example, be desirable to find conditions on the prescribed inflow data $f^{+}, f^{-}$which guarantee that the resulting Wigner function (when extended to the whole space) is physical.

For the time-dependent Wigner equation with inflow conditions, well-posedness has been studied for the linear ${ }^{14}$ and nonlinear ${ }^{15}$ cases. (See also Ref. 16, which studies inflow in the classical limit, and Ref. 17, where a strategy for coupling classical and quantum regimes is discussed.) One study has been made of the stationary Wigner equation with inflow conditions; ${ }^{18}$ a rather involved technical method was used to construct a solution. 
The quantity of interest to engineers is the stationary current as a function of applied voltage, the so-called $I-V$ curve. This explains our interest in the stationary problem. Our objective then is to show unique solvability of the stationary boundary value problem (BVP)

$$
v w_{x}-\Theta[V] w=0, \quad 0<x<L, \quad v \in \mathbb{R},
$$

subject to the BCs (1.5). In Ref. 11 Frensley analyzed a full discretization (upwind finite differences) of this stationary Wigner equation with inflow BCs, and there is numerical evidence that the problem is well-posed. A mathematical proof, however, has not yet been given.

At first glance (1.6) looks like a classical transport problem of the form

$$
T f_{x}-A f=0, \quad 0<x<L,
$$

for $f(x)$ in some Hilbert space, and $T \partial_{x}$ is the usual transport operator. For classical linear ${ }^{19}$ and nonlinear ${ }^{20,21}$ transport problems with inflow conditions, a great deal is known. In typical applications and in most of the mathematical analysis $A$ is a positive Fredholm operator, modeling the interaction of the "transport" particles with the medium in which they are diffusing. In (1.6), however, $-\Theta[V]$ describes the time-reversible interaction of the electrons with the system potential. Since it is skew-symmetric on $L^{2}\left(\mathbb{R}_{v}\right)$, standard techniques of generalized transport theory (see, e.g., Refs. 22 and 23) cannot be applied to the BVP of the Wigner equation, even in the linear case.

At this point it is in order to compare (1.6) with its classical counterpart, the BVP for the stationary Liouville equation:

$$
v w_{x}-V_{x} w_{v}=0, \quad 0<x<L, \quad v \in \mathbb{R} .
$$

Potential wells inside the domain $(0, L)$ give rise to closed particle trajectories, and hence the solution of (1.7) with inflow BCs (1.5) is in general not unique (see Ref. 17 for the linear problem, and Ref. 24 for the nonlinear case). The quantum picture is, however, different: Since bound states cannot be compactly supported, it is possible to "control" them through the inflow data.

The model we adopt in this paper, aside from being one dimensional and linear, is also discrete velocity. This may be considered either a preliminary step toward the analysis of the continuous-velocity model or an end in itself, since for numerics the velocity has to be discretized in any event (see Sec. II). In Sec. III we prove the well-posedness of the BVP problem for the discrete-velocity case, and discuss generalizations to the continuous-velocity case when including a cutoff for small velocities. Finally, in Sec. IV we find that the problem is not well-posed if $v$ $=0$ is included in the set of discrete velocities.

\section{VELOCITY DISCRETIZATION OF THE WIGNER EQUATION}

In Secs. III and IV we shall analyze the well-posedness of discrete velocity analogs of (1.6). In this section, we therefore discuss an example of how to obtain such a velocity semidiscretization. In Refs. 25 and 26 a spectral-collocation method (in velocity) of the time-dependent Wigner equation was studied. In order to obtain a simple discretization of $\Theta[V]$, the Wigner function is there approximated by a finite linear combination of trigonometric polynomials (in $v$ ), since they are (generalized) eigenfunctions of $\Theta[V]$ :

$$
\Theta[V] e^{i \eta v}=\delta V(x, \eta) e^{i \eta v},
$$

where

$$
\delta V(x, \eta)=i\left[V\left(x+\frac{\eta}{2}\right)-V\left(x-\frac{\eta}{2}\right)\right]
$$


is the symbol of $\Theta[V]$ [see (1.2b)]. Here we present a generalization of these techniques to infinitely many equidistant, discrete velocities.

The vector function $\mathbf{w}(x)=\left(w_{j}(x), j \in \mathbb{Z}\right)^{\top}$ denotes the discrete velocity Wigner function, where $w_{j}(x)$ is considered as an approximation to $w\left(x, v_{j}\right)$. Here, we choose the discrete velocities as $v_{j}=\left[\left(j-\frac{1}{2}\right) \pi\right] / \eta_{0}, j \in \mathbb{Z}$, where $\eta_{0}>0$ will be defined later on. With this choice we "skip" the discrete velocity $v=0$, as this would create analytical problems for the resulting BVP (Secs. III and IV).

In the sequel we consider $\mathbf{w}(x)$ as the sequence of the Fourier coefficients of the velocitytransformed function

$$
\hat{w}(x, \eta)=\sum_{j \in \mathbb{Z}} w_{j}(x) e^{-i v_{j} \eta}, \quad-\eta_{0} \leqslant \eta \leqslant \eta_{0}, \quad 0 \leqslant x \leqslant L,
$$

and conversely:

$$
w_{j}(x)=\frac{1}{2 \eta_{0}} \int_{-\eta_{0}}^{\eta_{0}} \hat{w}(x, \eta) e^{i \eta v_{j}} d \eta, \quad j \in \mathbb{Z} .
$$

Obviously $\hat{w}(x,.) \in L^{2}\left(-\eta_{0}, \eta_{0}\right)$ iff $\mathbf{w}(x) \in l^{2}(\mathbb{Z}) . \quad \eta_{0}$ gives the finite support (bandwidth) of $\hat{w}(x, \eta)$. In order to evaluate $\Theta[V]$ we extend $\hat{w}$ by 0 from $L^{2}\left(-\eta_{0}, \eta_{0}\right)$ to $L^{2}\left(\mathbb{R}_{\eta}\right)$. This corresponds to a trigonometric interpolation of $\mathbf{w}(x)$ on $\mathbb{R}_{v}$ :

$$
\widetilde{w}(x, v):=\frac{1}{2 \eta_{0}} \int_{-\eta_{0}}^{\eta_{0}} \hat{w}(x, \eta) e^{i \eta v} d \eta, \quad v \in \mathbb{R},
$$

and we have $\widetilde{w}\left(x, v_{j}\right)=w_{j}(x)$. This function $\widetilde{w}(x, v)$ is considered as a smooth approximation to $w(x, v)$. Since $\mathbf{w}(x) \in l^{2}(\mathbb{Z})$, this implies $\widetilde{w}(x,.) \in L^{2}\left(\mathbb{R}_{v}\right) \cap C^{\infty}$ and all $v$ derivatives decay at infinity:

$$
\left(\partial_{v}^{n} \widetilde{w}\right)(x, v) \rightarrow 0 \quad \text { for }|v| \rightarrow \infty, \quad n \in \mathbb{N}_{0} .
$$

Using (2.1) and (2.4) we calculate

$$
(\Theta[V] \widetilde{w})(x, v)=\frac{1}{2 \eta_{0}} \int_{-\eta_{0}}^{\eta_{0}} \delta V(x, \eta) \hat{w}(x, \eta) e^{i \eta v} d \eta
$$

If $V \in L^{\infty}(\mathbb{R})$ (which is the typical situation in semiconductor applications) we have $\Theta[V] \widetilde{w}(x,.) \in L^{2}\left(\mathbb{R}_{v}\right)$ with

$$
\|(\Theta[V] \widetilde{w})(x, .)\|_{2} \leqslant 2\|V\|_{\infty}\|\widetilde{w}(x, .)\|_{2} .
$$

When finally inserting (2.2) into (2.5) we get the desired discretization of $\Theta[V]$ for fixed $x$ $\in[0, L]:$

$$
\begin{gathered}
A(x): l^{2}(\mathbb{Z}) \rightarrow l^{2}(\mathbb{Z}), \\
(A(x) \mathbf{w})_{j}=\frac{1}{2 \eta_{0}} \int_{-\eta_{0}}^{\eta_{0}} \delta V(x, \eta)\left[\sum_{k \in \mathbb{Z}} w_{k} e^{i \eta\left(v_{j}-v_{k}\right)}\right] d \eta .
\end{gathered}
$$

For $V \in L^{\infty}(\mathbb{R})$ we have $A(x) \in B\left(l^{2}(\mathbb{Z})\right)$ with

$$
\|A(x)\| \leqslant 2\|V\|_{\infty} .
$$

We now proceed with a formal calculation to represent (2.7) as a discrete convolution [similar to the convolution representation (1.3) of $\Theta[V]]$ : 


$$
(A(x) \mathbf{w})_{j}=\sum_{k \in \mathbb{Z}} w_{k} a_{j-k}(x), \quad j \in \mathbb{Z},
$$

with

$$
a_{j}(x)=\frac{1}{2 \eta_{0}} \int_{-\eta_{0}}^{\eta_{0}} \delta V(x, \eta) \exp \left(\frac{i \pi j \eta}{\eta_{0}}\right) d \eta
$$

We remark that (2.9) is equivalent to (2.7) only under restrictive assumptions on the potential $V$. For the analysis of the discretized BVP in Sec. III we shall need $A(x) \in B\left(l^{2}(\mathbb{Z})\right)$. In the convolution form (2.9), the boundedness of $A(x)$ follows, e.g., if $\left(a_{j}, j \in \mathbb{Z}\right) \in l^{1}$. But for an arbitrary potential $V \in L^{\infty}$ the Fourier coefficients $\left(a_{j}\right)$ are usually not in $l^{1}$. Sufficient conditions for Fourier coefficients to be $l^{1}$ are listed, for example, in Sec. I.6 of Ref. 27.

\section{WELL-POSEDNESS OF THE BOUNDARY VALUE PROBLEM}

In this section we analyze the well-posedness of the discrete velocity analog of (1.6). The vector function $\mathbf{w}(x)=\left(w_{j}(x), j \in J\right)$ still denotes the discrete velocity Wigner function. The discrete velocities $v_{j} \in \mathbb{R}$ are assumed to be strictly increasing, i.e., $v_{j}<v_{j+1}$, and the index set $J \subset \mathbb{Z}$ might be finite or countably infinite. In the sequel we also assume $v_{j}>0$ for $j>0$ (i.e., $j \in J^{+}$ $:=J \cap \mathbb{N}$ ), and $v_{j}<0$ for $j<0$ [i.e., $j \in J^{-}:=J \cap(-\mathbb{N})$ ]; we set $v_{0}=0$, and generally assume in this section that $0 \notin J$. Note that, due to these assumptions on $v_{j}$, the discrete velocities cannot accumulate at zero; this fact will be important for our subsequent analysis.

Our stationary discrete velocity Wigner equation hence reads as

$$
T \mathbf{w}_{x}-A(x) \mathbf{w}=0, \quad 0<x<L,
$$

subject to the inflow BCs

$$
w_{j}(0)=f_{j}, \quad j \in J^{+}, \quad w_{j}(L)=f_{j}, \quad j \in J^{-},
$$

with a given sequence $\mathbf{f}=\left(f_{j}, j \in J\right)$. Here, $T=\operatorname{diag}\left(v_{j}\right)_{j \in J}$ is the diagonal matrix of the discrete velocities, and the real-valued matrix $A(x)$ is an appropriate semidiscretization (in $v$ ) of the operator $\Theta[V]$ for a given potential $V$; often the matrix $A(x)=\left(a_{j k}(x)\right)_{j, k \in J}$ will be a Toeplitz matrix [where $a_{j k}(x)=a_{j-k}(x)$, at least for an equidistant velocity discretization (see Sec. 2 of Ref. 26).

In the sequel we shall assume that the matrices $A(x)(0 \leqslant x \leqslant L)$ are skew-symmetric, reflecting the skew-symmetry of $\Theta[V]$. This is the key structural property that guarantees the unique solvability of the two-point BVP (3.1) and (3.2). If the discrete velocity $v_{0}=0$ is included in our model (i.e., if $0 \in J$ ) then (3.1), (3.2) is a differential-algebraic boundary value problem (DAEBVP) which behaves very differently from the BVP for an ordinary differential equation. Hence, in this section we will assume $0 \notin J$, and we discuss the zero velocity case in Sec. IV. Possible degeneracies when including zero as a discrete velocity in kinetic boundary value problems were already observed before: cf. Refs. 28 and 29, where stationary solutions to the discrete velocity Boltzmann equation were analyzed.

We analyze the BVP (3.1), (3.2) in the real Hilbert space $H:=l^{2}(J)$ with the natural inner product

$$
\langle\mathbf{w}, \mathbf{y}\rangle=\sum_{j \in J} w_{j} y_{j}
$$

$H$ may be decomposed as $H=H^{+} \oplus H^{-}$where $H^{ \pm}:=l^{2}\left(J^{ \pm}\right)$. We denote by $Q^{ \pm}$the restrictions of $H$ onto $H^{ \pm}$, i.e., $Q^{ \pm} \mathbf{w}=\mathbf{w}^{ \pm}$for any $\mathbf{w}=\left(\mathbf{w}^{+}, \mathbf{w}^{-}\right), \mathbf{w}^{ \pm} \in H^{ \pm}$. Let $P^{ \pm}$denote the projections defined by $P^{+} \mathbf{w}:=\left(\mathbf{w}^{+}, 0\right), P^{-} \mathbf{w}:=\left(0, \mathbf{w}^{-}\right)$; the embeddings $E^{ \pm}: H^{ \pm} \rightarrow H$ are defined by $E^{+} \mathbf{w}^{+}$ 
$:=\left(\mathbf{w}^{+}, 0\right), E^{-} \mathbf{w}^{-}:=\left(0, \mathbf{w}^{-}\right)$. One has the relations $P^{ \pm}=E^{ \pm} Q^{ \pm}$. We set $D:=T^{-1}, D$ being the diagonal operator $\operatorname{diag}\left(1 / v_{j}\right)$. Due to our assumptions on the $v_{j}$ we have $D \in B(H)$ (the bounded linear operators on $H$ ).

We assume $A$ to be an operator in $L^{1}((0, L), B(H))$ such that $A(x)$ is skew-symmetric for all $x \in[0, L]$. By a mild solution of the BVP $(3.1),(3.2)$ we mean a function $\mathbf{w} \in W^{1,1}((0, L), H)$ such that

$$
\mathbf{w}(x)=\mathbf{w}_{0}+\int_{0}^{x} D A\left(x^{\prime}\right) \mathbf{w}\left(x^{\prime}\right) d x^{\prime}
$$

is valid on $[0, L]\left[\right.$ where $\left.\mathbf{w}_{0}=\mathbf{w}(0)\right]$ and such that (3.2) is fulfilled, i.e.,

$$
\mathbf{w}^{+}(0)=\mathbf{f}^{+}, \quad \mathbf{w}^{-}(L)=\mathbf{f}^{-},
$$

where $\mathbf{f}=\left(\mathbf{f}^{+}, \mathbf{f}^{-}\right)$is given. By a classical solution of the BVP (3.1), (3.2) we mean a function $\mathbf{w} \in C^{1}([0, L], H)$ satisfying

$$
\mathbf{w}_{x}-D A(x) \mathbf{w}=0
$$

on $[0, L]$ such that $(3.4)$ is fulfilled.

We decompose a given skew-symmetric $A \in L^{1}((0, L), B(H))$ as

$$
A(x)=\left(\begin{array}{ll}
A^{++} & A^{+-} \\
A^{-+} & A^{--}
\end{array}\right)=-A^{*}(x)
$$

with $A^{++}:=Q^{+} A E^{+} \in B\left(H^{+}\right), A^{+-}:=Q^{+} A E^{-} \in B\left(H^{-}, H^{+}\right), A^{-+}:=Q^{-} A E^{+} \in B\left(H^{+}, H^{-}\right), A^{--}$ $:=Q^{-} A E^{-} \in B\left(H^{-}\right)$. Also, one has

$$
D=\left(\begin{array}{cc}
D^{+} & 0 \\
0 & D^{-}
\end{array}\right), \quad|D|=\left(\begin{array}{cc}
D^{+} & 0 \\
0 & -D^{-}
\end{array}\right),
$$

where $D^{ \pm}:=\operatorname{diag}\left(1 / v_{j}\right)_{j \in J^{ \pm}}$. We get $|D| \geqslant 0$ in the Hilbert space sense, i.e., $\langle|D| \mathbf{w}, \mathbf{w}\rangle \geqslant 0$ for every $\mathbf{w} \in H$.

Crucial for our analysis is the following transformation of the BVP (3.1), (3.2): We introduce a vector $\mathbf{z}$ by $\mathbf{w}=: \sqrt{|D|} \mathbf{z}$, and $\mathbf{z} \in H$ implies $\mathbf{w} \in H$. Then the transformed problem has the form

$$
\begin{gathered}
\mathbf{z}_{x}-B(x) \mathbf{z}=0, \quad 0<x<L, \\
\mathbf{z}^{+}(0)=\sqrt{|D|^{-1}} \mathbf{f}^{+}=: \mathbf{g}^{+}, \quad \mathbf{z}^{-}(L)=\sqrt{|D|^{-1}} \mathbf{f}^{-}=: \mathbf{g}^{-},
\end{gathered}
$$

where $\mathbf{g}=\left(\mathbf{g}^{+}, \mathbf{g}^{-}\right)$are the transformed inflow data, and we shall assume $\mathbf{g} \in H$. The operator $B$ is defined as $B(x):=\sqrt{|D|}{ }^{-1} D A(x) \sqrt{|D|}$, and the assumptions on $A$ imply $B \in L^{1}((0, L), B(H))$ since $\sqrt{|D|} \in B(H)$. We may write $B(x)$ in the form

$$
B(x)=\left(\begin{array}{cc}
\sqrt{D^{+}} A^{++}(x) \sqrt{D^{+}} & \sqrt{D^{+}} A^{+-}(x) \sqrt{-D^{-}} \\
-\sqrt{-D^{-}} A^{-+}(x) \sqrt{D^{+}} & -\sqrt{-D^{-}} A^{--}(x) \sqrt{-D^{-}}
\end{array}\right) .
$$

Hence we have $\left\langle A \mathbf{w}, \mathbf{w}^{\prime}\right\rangle=\left\langle\operatorname{sign}(D) B \mathbf{z}, \mathbf{z}^{\prime}\right\rangle$ for all $\mathbf{z}={\sqrt{|D|^{-1}}}^{-\mathbf{w}}$ and $\mathbf{z}^{\prime}={\sqrt{|D|^{-1}}}^{\prime} \in H$, and (3.6) gives

$$
B^{++}(x)=-B^{++}(x)^{*}, \quad B^{--}(x)=-B^{--}(x)^{*}, \quad B^{+-}(x)=B^{-+}(x)^{*} .
$$

Our next goal is to reformulate the BVP (3.8), (3.9) as an initial value problem (IVP) together with an operator equation to calculate the outflow data in terms of the given inflow data. To this end let us consider the IVP 


$$
\begin{gathered}
\mathbf{z}_{x}-B(x) \mathbf{z}=0, \quad 0<x<L, \\
\mathbf{z}(0)=\mathbf{z}_{0} \in H .
\end{gathered}
$$

Lemma 3.1: If $B \in L^{1}((0, L), B(H))$ then the IVP (3.8), (3.12) has a unique mild solution $\mathbf{z}$ $\in W^{1,1}((0, L), H)$, and there exists a unique strongly continuous propagator $U\left(x, x^{\prime}\right) \in B(H) \forall 0 \leqslant x, x^{\prime} \leqslant L$. It satisfies

$$
F^{\prime}(x)-B(x) F(x)=0, \quad G^{\prime}(x)+G(x) B(x)=0, \quad F^{* \prime}(x)-F^{*}(x) B^{*}(x)=0
$$

almost everywhere on $(0, L)$, where

$$
F(x):=U(x, 0), \quad G(x):=F^{-1}(x)=U(0, x),
$$

and $F(0)=G(0)=F^{*}(0)=I$.

Proof: The result follows by a simple extension of the first theorems of Sec. 5.1 of Ref. 30. The identities (3.13) follow easily from the mild version of the differential equation and the definition of $F(x)$ and $G(x)$.

We are now in the position to reformulate the BVP (3.8), (3.9) using the propagator $U$ of the IVP (3.8), (3.12). Since the inflow data $\mathbf{z}^{+}(0)=\mathbf{g}^{+}, \mathbf{z}^{-}(L)=\mathbf{g}^{-}$are given we can get the solution of the BVP (3.8), (3.9) by

$$
\mathbf{z}(x)=U(x, 0)\left(\begin{array}{l}
\mathbf{g}^{+} \\
\mathbf{h}^{-}
\end{array}\right)=U(x, L)\left(\begin{array}{l}
\mathbf{h}^{+} \\
\mathbf{g}^{-}
\end{array}\right), \quad 0 \leqslant x \leqslant L,
$$

if the a priorily unknown outflow data $\mathbf{z}^{-}(0)=\mathbf{h}^{-}, \mathbf{z}^{+}(L)=\mathbf{h}^{+}$could be determined. The idea is to calculate $\mathbf{h}^{+}$from (3.14) by eliminating $\mathbf{h}^{-}$. The vectors $\mathbf{h}^{ \pm}$satisfy

$$
\left(\begin{array}{c}
\mathbf{h}^{+} \\
0
\end{array}\right)=P^{+} U(L, 0)\left(\begin{array}{l}
\mathbf{g}^{+} \\
\mathbf{h}^{-}
\end{array}\right), \quad\left(\begin{array}{c}
0 \\
\mathbf{h}^{-}
\end{array}\right)=P^{-} U(0, L)\left(\begin{array}{l}
\mathbf{h}^{+} \\
\mathbf{g}^{-}
\end{array}\right) .
$$

From (3.15) one gets by insertion (using the notation from Lemma 3.1)

$$
\left(\begin{array}{c}
\mathbf{h}^{+} \\
0
\end{array}\right)=P^{+} F(L)\left[\left(\begin{array}{c}
\mathbf{g}^{+} \\
0
\end{array}\right)+P^{-} G(L)\left(\begin{array}{l}
\mathbf{h}^{+} \\
\mathbf{g}^{-}
\end{array}\right)\right],
$$

and when solving for $\mathbf{h}^{+}$,

$$
\left[I-P^{+} F(L) P^{-} G(L) P^{+}\right]\left(\begin{array}{c}
\mathbf{h}^{+} \\
0
\end{array}\right)=P^{+} F(L)\left[\left(\begin{array}{c}
\mathbf{g}^{+} \\
0
\end{array}\right)+P^{-} G(L)\left(\begin{array}{c}
0 \\
\mathbf{g}^{-}
\end{array}\right)\right]=l(\mathbf{g}) .
$$

In (3.17) the right-hand side $l(\mathbf{g})$ is datum. Introducing $K:=\left[I-P^{+} F(L) P^{-} G(L) P^{+}\right]$one gets from (3.16) an operator equation for $\mathbf{h}^{+}$, namely

$$
[I-K]\left(\begin{array}{c}
\mathbf{h}^{+} \\
0
\end{array}\right)=l(\mathbf{g}) .
$$

If (3.18) is uniquely solvable then, by inserting the solution $\mathbf{h}^{+}$into the second equation of (3.15), one can determine $\mathbf{h}^{-}$. And one then gets the unique solution $\mathbf{z}(x)$ of the BVP (3.8), (3.9) by either formula of (3.14). Thus, our goal is to show that (3.18) is uniquely solvable in $H$.

One can write

$$
K=\left(\begin{array}{cc}
0 & F(L)^{+-} \\
0 & 0
\end{array}\right)\left(\begin{array}{cc}
0 & 0 \\
G(L)^{-+} & 0
\end{array}\right)=\left(\begin{array}{cc}
F(L)^{+-} G(L)^{-+} & 0 \\
0 & 0
\end{array}\right) .
$$


The unique solvability of Eq. (3.18) is now implied in the following lemma, which exhibits the main structural property of our problem.

Lemma 3.2: If $B \in L^{1}((0, L), B(H))$ then $K \leqslant 0$ (in the Hilbert space sense).

Proof: Define the matrix operator $\widetilde{G}(x)$ on $H$ by

$$
\widetilde{G}(x):=\left(\begin{array}{cc}
G(x)^{++} & -G(x)^{+-} \\
-G(x)^{-+} & G(x)^{--}
\end{array}\right), \quad 0<x<L .
$$

By using (3.11) and (3.13) one sees that the operator differential equation $\widetilde{G}^{\prime}(x)-\widetilde{G}(x) B^{*}(x)$ $=0$ is satisfied a.e. on $(0, L)$ which is the same equation that is fulfilled by $F^{*}(x)$ [see (3.13)]; since $\widetilde{G}(0)=F^{*}(0)=I$ holds, $\widetilde{G}(x)=F^{*}(x)$ follows a.e. on $(0, L)$. Since $F(x)$ [and hence $F^{*}(x)$ ] and $G(x)$ [and hence $\widetilde{G}(x)]$ are strongly continuous in $x$ on $[0, L]$ this implies that $\widetilde{G}(x)$ $=F^{*}(x)$ for all $x \in[0, L]$. Thus, we see that

$$
G(L)^{-+}=-\widetilde{G}(L)^{-+}=-\left(F^{*}(L)\right)^{-+}=-\left(F(L)^{+-}\right)^{*} .
$$

This in turn implies

$$
K^{++}=F(L)^{+-} G(L)^{-+}=-F(L)^{+-}\left(F(L)^{+-}\right)^{*} \leqslant 0 .
$$

From Lemma 3.2 we immediately deduce

Theorem 3.3: Assume $0 \notin J, \mathbf{g}=\left(\mathbf{g}^{+}, \mathbf{g}^{-}\right) \in H$, and let $A(x) \in B(H)$ be skew-symmetric for all $x \in[0, L]$. Then one has:

(a) If $A \in L^{1}((0, L), B(H))$ the BVP (3.8), (3.9) has a unique mild solution $\mathbf{z} \in W^{1,1}((0, L), H)$.

(b) If $A(x)$ is strongly continuous in $x$ on $[0, L]$ and uniformly bounded in the norm of $B(H)$ on $[0, L]$, then the solution from (a) is a classical solution, i.e., $\mathbf{z} \in C^{1}([0, L], H)$.

Proof: The assumptions on $A$ imply that $B \in L^{1}((0, L), B(H))$. Lemma 3.2 and the selfadjointness of the bounded operator $K$ imply that $I-K$ is invertible with a bounded inverse; this shows the unique solvability of the BVP.

To prove (b) we only have to show that a mild solution $\mathbf{z} \in L^{1}((0, L), H)$ is in $C^{1}([0, L], H)$. Since $\mathbf{z}$ is in $W^{1,1}((0, L), H)$ it is also in $C([0, L], H)$. Since

$$
\mathbf{z}(x)=\mathbf{z}_{0}+\int_{0}^{x} B\left(x^{\prime}\right) \mathbf{z}\left(x^{\prime}\right) d x^{\prime}
$$

is satisfied on $[0, L]$, it is enough to show that the mapping $x \rightarrow B(x) \mathbf{z}(x)=: \mathbf{u}(x)$ is in $C([0, L], H)$. Let $x \in[0, L]$ be fixed, and let $x_{n} \rightarrow x$ (for $n \rightarrow \infty$ ), then it follows that for $n \rightarrow \infty$ we have $\mathbf{u}(x)$ $-\mathbf{u}\left(x_{n}\right)=\left[B(x)-B\left(x_{n}\right)\right] \mathbf{z}(x)+B\left(x_{n}\right)\left[\mathbf{z}(x)-\mathbf{z}\left(x_{n}\right)\right] \rightarrow 0$ since $B(x)$ is strongly continuous and uniformly bounded, and $\mathbf{z}$ is continuous.

With the transformation $\mathbf{w}=\sqrt{|D|} \mathbf{z}$ Theorem 3.3 immediately translates into a result for our original BVP (3.1), (3.2). We remark that $\mathbf{z} \in H$ iff $\mathbf{w} \in \widetilde{H}:=l^{2}\left(J ;\left|v_{j}\right|\right)$. Here $\left|v_{j}\right|$ denotes a weight function, i.e., we endow $\widetilde{H}$ with the inner product

$$
\langle\langle\mathbf{w}, \mathbf{y}\rangle\rangle=\sum_{j \in J}\left|v_{j}\right| w_{j} y_{j}
$$

[if $T=\operatorname{diag}\left(v_{j}\right)_{j \in J}$ is bounded, then the Hilbert spaces $H$ and $\widetilde{H}$ coincide and the two norms are equivalent]. Such weighted spaces are typical for kinetic BVPs [see Refs. 31 and 13, where $L^{2}\left(\mathbb{R}_{v} ;|v|\right)$ is the appropriate space for the (nondiscrete) boundary data]. 
Proposition 3.4: Assume $0 \notin J, \mathbf{f}=\left(\mathbf{f}^{+}, \mathbf{f}^{-}\right) \in \widetilde{H}$, and let $A(x) \in B(H)$ be skew-symmetric for all $x \in[0, L]$. Then one has:

(a) If $A \in L^{1}((0, L), B(H))$ the $B V P$ (3.1), (3.2) has a unique mild solution $\mathbf{w} \in W^{1,1}((0, L), \widetilde{H})$. Also, $T \mathbf{w}_{x} \in L^{1}((0, L), H)$.

(b) If $A(x)$ is strongly continuous in $x$ on $[0, L]$ and uniformly bounded in the norm of $B(H)$ on $[0, L]$, then the solution from (a) is a classical solution, i.e., $\mathbf{w} \in C^{1}([0, L], \widetilde{H})$. Also, $T \mathbf{w}_{x}$ $\in C([0, L], H)$.

Proof: The assertions on $T \mathbf{w}_{x}$ follow from $\widetilde{H} \subset H$ and the discretized Wigner equation (3.1).

We shall now outline an alternative well-posedness proof for the BVP (3.1), (3.2). This approach complements the above result as it will require different assumptions on $\mathbf{f}$ and $A$. This strategy was suggested by the anonymous referee and it is more closely related to known techniques in generalized transport theory. ${ }^{23,32}$

We now consider the BVP (3.1), (3.4) for $w \in X:=L^{2}\left((0, L) \times \mathbb{R}_{v} ; d x d \mu\right)$, where $d \mu$ is a positive Radon measure satisfying $\mu\{0\}=0$. In our discrete-velocity case we have

$$
d \mu=\sum_{j \in J} \delta\left(v-v_{j}\right)
$$

with $v_{j}<v_{j+1}$ and $0 \notin J$. We adopt here a notation that equally applies to discrete and continuous velocities, as we shall comment on the latter case at the end of this section. $A(x)$ is either the pseudo-differential operator $\Theta[V]$ (in the continuous velocity case where $d \mu=d v$ ) or its velocity discretization. In either case we shall assume that $A(x)$ is skew-symmetric and

$$
A \in L^{\infty}\left((0, L), B\left(L^{2}\left(\mathbb{R}_{v} ; d \mu\right)\right)\right),
$$

and hence $A \in B(X)$.

In this second approach we shall assume inflow boundary data

$$
\left(f^{+}, f^{-}\right) \in Y:=L^{2}\left(R_{v} ; \min (1,|v|) d \mu\right),
$$

which is the appropriate space for the boundary traces: by Theorem 3 of Ref. $31, f^{ \pm} \in Y$ can be "lifted" to a function $f(x, v)$ with $f, v f_{x} \in X$. Hence, the inhomogeneous BVP (3.1), (3.4) and the following BVP for $y \in X$ are equivalent as far as existence and uniqueness is concerned:

$$
v y_{x}-A(x) y=g(x, v),
$$

with homogeneous boundary conditions

$$
y^{+}(0)=0, \quad y^{-}(L)=0 .
$$

Here we set $y=w-f$ and $g=A(x) f-v f_{x} \in X$.

The free-streaming operator $L=v \partial_{x}$ of (3.23a) is defined on

$$
\mathcal{D}(L)=\left\{y \in X \mid v y_{x} \in X, y^{+}(0)=0, y^{-}(L)=0\right\} .
$$

$L$ generates on $X$ a $C_{0}$ semigroup of contractions. $0 \notin \sigma(L)$ iff $v=0$ does not belong to the set of velocities (i.e., $\mu\{|v|<\varepsilon\}=0$ for some $\varepsilon>0$ ). In the discrete velocity case we assumed $0 \notin J$ and hence $L$ is invertible. Therefore (3.23) is equivalent to

$$
y-L^{-1}(A(x) y)=L^{-1} g(x, v),
$$

with the BCs (3.23b).

Lemma 3.5: $L^{-1}$ is compact on $X$ iff the discrete velocities $\left\{v_{j}, j \in J\right\}$ do not accumulate in $\mathbb{R}_{v}$. (Such an accumulation would anyhow not be relevant in practical cases.) 
Proof: For $y \in X$ we have

$$
\left(L^{-1} y\right)\left(x, v_{j}\right)= \begin{cases}\frac{1}{\left|v_{j}\right|} \int_{0}^{x} y\left(\xi, v_{j}\right) d \xi, & j>0 \\ \frac{1}{\left|v_{j}\right|} \int_{x}^{L} y\left(\xi, v_{j}\right) d \xi, & j<0 .\end{cases}
$$

In the case of finitely many velocities $L^{-1}$ is clearly compact. For infinitely many velocities without an accumulation point we decompose $L^{-1}$ :

$$
L^{-1}=P_{\tilde{v}} L^{-1}+\left(I-P_{\tilde{v}}\right) L^{-1},
$$

where $P_{\tilde{v}}$ is the projection from $L^{2}\left(\mathbb{R}_{v} ; d \mu\right)$ onto the (finitely many) velocities $\left|v_{j}\right|<\widetilde{v}$. The first summand of (3.26) is compact. The norm of the second summand is bounded by $\widetilde{v}^{-1}$ and can thus be made arbitrarily small by choosing $\widetilde{v}$ large enough. Hence, $L^{-1}$ and $L^{-1} A$ are compact.

If the discrete velocities $\left\{v_{j}\right\}$ accumulate (e.g., as $j \rightarrow \infty$ ), $L^{-1}$ is not compact: Consider the bounded squence $\left\{y^{n}, n \in \mathbb{N}\right\}$ with $y^{n}\left(x, v_{j}\right)=\delta_{j}^{n}$ (const in $x$ ), which does not have a convergent subsequence.

With this result we have the second well-posedness result for the BVP (3.1), (3.4):

Theorem 3.6: Assume that the velocities $\left\{v_{j}, j \in J\right\}$ do not accumulate in $\mathbb{R}_{v}$ and that $0 \notin J$. Let $A(x)$ be skew-symmetric for all $x \in[0, L]$ and let $A \in L^{\infty}\left((0, L), B\left(L^{2}\left(\mathbb{R}_{v} ; d \mu\right)\right)\right)$, and $\left(f^{+}, f^{-}\right) \in Y$. Then, (3.1), (3.4) has a unique solution $w \in X$. Also, $v w_{x} \in X$.

Proof: Due to Lemma 3.5 the Fredholm alternative applies to (3.24) and any solution $y \in X$ of (3.24) also satisfies $y \in \mathcal{D}(L)$. It remains to show that the homogeneous version of (3.23) (i.e., with $g=0)$ admits only the trivial solution $y=0$.

We multiply (3.23a) by $y$ and integrate over $(0, L) \times \mathbb{R}_{v}$ to obtain

$$
0=\int_{0}^{L} \int_{\mathbb{R}} v\left(y(x, v)^{2}\right)_{x} d \mu d x=\int_{0}^{\infty} v(y(L, v))^{2} d \mu-\int_{-\infty}^{0} v(y(0, v))^{2} d \mu,
$$

where we first used the skew-symmetry of $A(x)$ and then the BCs (3.23b). This implies $y^{-}(0)$ $=0$ and $y^{+}(L)=0$. Using the initial condition $y\left(0, v_{j}\right)=0, j \in J$ or $\mathbf{z}(0)=0$ for the IVP (3.8) implies $\mathbf{z} \equiv 0$ and $y \equiv 0$.

We finish this section with some remarks:

(1) The motivation for the assumptions on $A(x)$ stem directly from the continuous velocity case: There the pseudodifferential operator $A(x)=\Theta[V](x)$ in the Wigner equation has the explicit form

$$
A(x) w=i \mathcal{F}^{-1}\left\{[V(x+. / 2)-V(x-. / 2)] \mathcal{F}_{w}\right\}
$$

for $w \in L^{2}\left(\mathbb{R}_{v}\right)$ where $\eta \rightarrow V(x \pm \eta / 2)$ is a multiplication operator in the dual Fourier variable $\eta ; \mathcal{F}$ denotes the Fourier transform in the $v$ variable.

Assuming $V \in L^{\infty}\left(\mathbb{R}_{x}\right)$ one sees that $A(x) \in B\left(L^{2}\left(\mathbb{R}_{v}\right)\right)$ and $\|A(x)\| \leqslant 2\|V\|_{\infty}$. If the given potential decays sufficiently at infinity, $A(x)$ even regularizes:

$$
\|A(x)\|_{L^{2}\left(\mathbb{R}_{v}\right) \rightarrow H^{1}\left(\mathbb{R}_{v}\right)} \leqslant C\|(1+|x|) V\|_{\infty},
$$

with some constant $C$ that is independent of $0<x<L$.

Furthermore, using Lebesgue's dominated convergence theorem it is easy to see that $A(x)$ is strongly continuous on $[0, L]$. If the potential $V$ is discontinuous at (at least) one point in $[0, L]$, then $A(x)$ is discontinuous for every $x \in[0, L]$ in the uniform operator topology; this is easily seen, e.g., for a step potential modeling tunneling in semiconductor devices.

(2) Theorem 3.3 equally applies for the continuous-velocity case when one uses a velocity cutoff in the stationary Wigner equation in the vicinity of zero. For example, it applies to the 
following modification of (1.6):

$$
\zeta(v) w_{x}-\Theta[V] w=0,
$$

with a cutoff function $\zeta(v)=v$ for $|v|>\varepsilon>0$ and $|\zeta(v)| \geqslant \varepsilon$ elsewhere. Then, $T^{-1}=1 / \zeta(v)$ is again a bounded operator on $L^{2}\left(\mathbb{R}_{v}\right)$.

This velocity truncation is rather a technical restriction that could (possibly) be overcome in the future. We remark that such a cutoff for small velocities is frequently encountered in stationary kinetic problems: cf. Ref. 33, for example, where the steady Boltzmann equation is studied.

Lemma 3.5 does not carry over to the continuous-velocity case, since $L^{-1}$ is then not compact. However, if the potential $V$ decays such that $(1+|x|) V(x) \in L^{\infty}\left(\mathbb{R}_{x}\right)$, compactness in velocity direction (and hence a result analogous to Theorem 3.6) can be obtained from a decomposion similar to (3.26):

$$
\frac{1}{\zeta(v)} A(x)=P_{\tilde{v}} \frac{1}{\zeta(v)} A(x)+\left(I-P_{\widetilde{v}}\right) \frac{1}{\zeta(v)} A(x) .
$$

Here, the first operator on the right-hand side is compact due to the compact imbedding $H^{1}(-\widetilde{v}, \widetilde{v}) \rightarrow L^{2}(-\widetilde{v}, \widetilde{v})$ [cf. (3.27)], and the second operator can be made arbitrarily small.

(3) Our method and Theorem 3.3 apply also to symmetric velocity discretizations of the Liouville equation (1.7), showing that the discretized version of the inflow BVP (1.7), (1.5) is uniquely solvable. In the limit of continuous velocities, however, it is clear that no propagator $U\left(x, x^{\prime}\right)$ (cf. Lemma 3.1) can exist because of the characteristics intersecting the $x$ axis (unless $V$ $=$ const).

Similarly, the second approach (Theorem 3.6) would not work either for the classical case as $A(x)=V_{x} \partial_{v}$ is then unbounded.

(4) The original problem (1.6) - the stationary Wigner equation with continuous velocities-still poses additional analytical problems since here $T^{-1} A(x)$ is unbounded on $L^{2}\left(\mathbb{R}_{v}\right)$. This case will eventually be treated in a forthcoming paper. To this end (3.18) with $K \leqslant 0$ seems to be the crucial structural property, since it is independent of $\varepsilon$ in the above cutoff function $\zeta$.

\section{ZERO AS A DISCRETE VELOCITY}

In this section we shall analyze the discrete velocity Wigner equation

$$
T \mathbf{w}_{x}-A(x) \mathbf{w}=0, \quad 0<x<L,
$$

for $\mathbf{w}(x)=\left(w_{j}(x), j \in J\right)^{\top}$ in the case $0 \in J$. Again we prescribe inflow BCs

$$
\begin{aligned}
& \mathbf{w}^{+}(0)=\mathbf{f}^{+}, \\
& \mathbf{w}^{-}(L)=\mathbf{f}^{-} .
\end{aligned}
$$

The model now includes the discrete velocity $v_{0}=0$, hence (4.1) is a linear differential-algebraic equation (DAE, see, e.g., Refs. 34 and 35). Frensley already mentioned in Ref. 11 that one should "avoid" this zero velocity in the numerical discretization of the stationary Wigner equation on a slab. In this section we shall analyze the algebraic reasons for it. To illustrate the problems encountered here, we will first consider an example of dimension $m:=|J|=3$ with a constant matrix $A$ :

Example 4.1:

$$
T \mathbf{w}_{x}-\left(\begin{array}{ccc}
0 & -a_{1,0} & -a_{1,-1} \\
a_{1,0} & 0 & -a_{0,-1} \\
a_{1,-1} & a_{0,-1} & 0
\end{array}\right) \mathbf{w}=0, \quad 0<x<L,
$$


with $T=\operatorname{diag}\left(v_{1}, 0, v_{-1}\right), \mathbf{w}=\left(w_{1}, w_{0}, w_{-1}\right)^{\top}$, and the BCs

$$
w_{1}(0)=f_{1}, \quad w_{-1}(L)=f_{-1} .
$$

In order to avoid trivialities we assume that $a_{1,0}, a_{0,-1} \neq 0$.

As the second row of (4.3) - the algebraic constraint-does not involve $w_{0}$, one has to differentiate algebraic constraints twice in order to express $\left(w_{0}\right)_{x}$ as a function of $\mathbf{w}$. Hence, the index of (4.3) is 2 .

The constant-coefficient DAE (4.3) is called tractable ${ }^{34}$ or solvable if $^{35}$ if the determinant

$$
\operatorname{det}(\lambda T-A)=\lambda\left(v_{1} a_{0,-1}^{2}+v_{-1} a_{1,0}^{2}\right)
$$

does not vanish identically for $\lambda \in \mathbb{C}$. First we consider the case $v_{1} a_{0,-1}^{2}+v_{-1} a_{1,0}^{2} \neq 0$. Then (4.3) is easily seen to be equivalent to

$$
\begin{gathered}
\left(w_{1}\right)_{x}=0, \\
a_{0,-1} w_{-1}=a_{1,0} w_{1}, \\
a_{0,-1} w_{0}=a_{1,-1} w_{1} .
\end{gathered}
$$

And, obviously, only one BC can be specified for (4.3) in this case.

In the nontractable case, i.e., for $v_{1} a_{0,-1}^{2}+v_{-1} a_{1,0}^{2}=0$, the three rows of (4.3) are linearly dependent (after differentiating the second row). Hence, there are more than countably many solutions to the BVP (4.3), (4.4) in this case:

$$
\begin{gathered}
w_{1}(x)=f_{1} \exp \left(-\frac{a_{1,-1} a_{1,0}}{v_{1} a_{0,-1}}\right)-\frac{a_{1,0}}{v_{1}} \int_{0}^{x} w_{0}\left(x^{\prime}\right) \exp \left(-\frac{a_{1,-1} a_{1,0}}{v_{1} a_{0,-1}}\left(x-x^{\prime}\right)\right) d x^{\prime}, \\
w_{-1}(x)=\frac{a_{1,0}}{a_{0,-1}} w_{1}(x),
\end{gathered}
$$

and $w_{0}$ is arbitrary up to the constraint $w_{-1}(L)=f_{-1}$.

We summarize the situation of Example 3.1 in

Proposition 4.2: For any matrices $T$ and $A$ of the above structure, the DAE (4.3) is not well-posed if two independent BCs (4.4) are prescribed.

We now turn to the general situation (4.1), (4.2). To avoid technical difficulties, we confine ourselves to the finite dimensional case $(m<\infty)$ and we assume $A(x)$ to be sufficiently smooth in $x \in[0, L]$.

In the sequel we shall assume that (4.1) is solvable (see Sec. 2.4.1 of Ref. 35 for the definition in the variable coefficient case). Otherwise (4.1) would not have a solution $\mathbf{w}(x), 0<x<L$ that is uniquely determined by fixing the solution $\mathbf{w}\left(x_{0}\right)$ at one point $x_{0}$.

In the variable coefficient case there is no simple characterization of solvability [like $\operatorname{det}(\lambda T$ $-A) \not \equiv 0$ for constant coefficients], but we can give a sufficient criterion. First, we remark that (4.1) can be written in Hessenberg form of size 2, i.e.,

$$
\left(\begin{array}{cc}
I_{m-1} & 0 \\
0 & 0
\end{array}\right) \mathbf{w}_{x}-\left(\begin{array}{cc}
\widetilde{A}_{11}(x) & \widetilde{A}_{12}(x) \\
\widetilde{A}_{21}(x) & 0
\end{array}\right) \mathbf{w}=0, \quad 0<x<L,
$$

if the constraint

$$
\widetilde{A}_{21}(x) \widetilde{A}_{12}(x)=-\sum_{j \neq 0} \frac{a_{j 0}^{2}(x)}{v_{j}} \neq 0 \quad \forall x \in[0, L]
$$


is satisfied. Here, $\widetilde{A}_{21}(x)$ and $\widetilde{A}_{12}(x)$ are, respectively, $1 \times(m-1)$ and $(m-1) \times 1$ matrices.

Propsition 4.3 (Sec. 2.4 .2 of Ref. 35): If (4.1) is in Hessenberg form of size 2, then it is solvable and has index 2.

A simple calculation gives

$$
\operatorname{det}(\lambda T-A(x))=\lambda^{m-2}\left(\sum_{j \neq 0} \frac{a_{j 0}^{2}(x)}{v_{j}}\right) \prod_{j \neq 0} v_{j}+\text { lower order terms }(\text { in } \lambda)
$$

[see (4.5)]. Hence, the Hessenberg condition (4.6) also implies the local (in $x$ ) regularity of the matrix pencil $\lambda T-A(x)$.

Next we discuss the index of the DAE (4.1). Since $T$ is constant in $x$, it has constant rank, and the differentiation procedure (of the algebraic constraint) can be used to define the index of (4.1), i.e., the minimum number of differentiations necessary to express $\mathbf{w}_{x}$ explicitly as a continuous function of $\mathbf{w}$ and $x$ (see Sec. 2.4.1 of Ref. 35). In (4.1) the algebraic constraint has the form

$$
\sum_{j \in J} a_{0, j}(x) w_{j}=0
$$

Since $a_{0,0}(x) \equiv 0,(4.1)$ has an index $k \geqslant 2$ (if it is solvable). The index can indeed exceed 2 (e.g., for $m=4$, the index 4 is possible).

Since the differentiation procedure works for (4.1) it can be further shown (see Sec. 2.4.2 of Ref. 35) that (4.1) is (analytically) equivalent to a decoupled system in standard canonical form:

$$
\left(\begin{array}{cc}
I_{m-s} & 0 \\
0 & N(x)
\end{array}\right) \mathbf{y}_{x}-\left(\begin{array}{cc}
C(x) & 0 \\
0 & I_{s}
\end{array}\right) \mathbf{y}=0, \quad 0<x<L,
$$

where $\mathbf{w}=R(x) \mathbf{y}$ is a change of coordinates with a smooth, nonsingular matrix $R(x)$, and we partition $\mathbf{y}=\left(\mathbf{y}_{1}, \mathbf{y}_{2}\right)^{\top} . N(x)$ is a strictly lower triangular, square matrix of size $s \geqslant k$ with nilpotency $k$. One easily verifies that the second equation of (4.8),

$$
N(x)\left(\mathbf{y}_{2}\right)_{x}-\mathbf{y}_{\mathbf{2}}=0
$$

only has the trivial solution $\mathbf{y}_{\mathbf{2}}(x) \equiv 0$, and hence, no BCs may be specified for $\mathbf{y}_{\mathbf{2}}$. A necessary condition for the unique solvability of the first equation of (4.8) is to specify $m-s$ BCs.

We therefore conclude that only $m-s(\leqslant m-k \leqslant m-2)$ BCs may be prescribed for (4.8) or, equivalently, (4.1). The $m-1$ BCs (4.2) hence make the system overdetermined.

We summarize in

Theorem 4.4: (a) If the DAE (4.1) is not solvable (in the sense of Sec. 2.4.1 of Ref. 35), solutions of (4.1), (4.2) cannot be unique.

(b) If the DAE (4.1) is solvable, the BCs (4.2) are overdetermined and hence make the BVP (in general) unsolvable.

\section{ACKNOWLEDGMENTS}

A. A. was partially supported by Grant No. ERBFMRXCT970157 (TMR-Network) from the EU, the bilateral DAAD-NSF Program (315/PPP/ru-ab), and the DFG under Grant-No. MA 1662/ 1-2. The authors wish to express their gratitude to the Erwin Schrödinger-Institute, Vienna and the Department of Physics, Harvard University and to Professor Arthur Jaffe for their hospitality, while the research for this paper was carried out. P.F.Z. would like to express his appreciation to the Dipartimento di Matematica Applicata, Università di Firenze, where a portion of this manuscript was prepared. 
${ }^{1}$ E. Wigner, "On the quantum correction for thermodynamic equilibrium," Phys. Rev. 40, 749-759 (1932).

${ }^{2}$ P. A. Markowich, "On the equivalence of the Schrödinger equation and the quantum Liouville equation," Math. Methods Appl. Sci. 11, 459-469 (1989).

${ }^{3}$ A. Arnold and F. Nier, "Numerical analysis of the deterministic particle method applied to the Wigner equation," Math. Comput. 58, 645-669 (1992).

${ }^{4}$ K. Imre, E. Özizmir, M. Rosenbaum, and P. F. Zweifel, “Wigner method in quantum statistical mechanics,” J. Math. Phys. 8, 1097-1108 (1967).

${ }^{5}$ P. Carruthers and F. Zachariasen, "Quantum collision theory with phase space distributions," Rev. Mod. Phys. 55, 245-285 (1983).

${ }^{6}$ M. C. Teich and B. F. A. Saleh, "Squeezed and antibunched light," Phys. Today 43, 26-34 (1990).

${ }^{7}$ M. di Toro, U. Lombardo, and G. Russo, "Small oscillations in a nuclear Vlasov fluid," Nuovo Cimento A 87, 174-189 (1985).

${ }^{8}$ P. F. Zweifel and B. Toomire, "Boundary conditions in quantum transport theory," Transp. Theory Stat. Phys. 26, 629-636 (1997).

${ }^{9}$ N. Kluksdahl, A. M. Kriman, D. K. Ferry, and C. Ringhofer, "Self-consistent study of the resonant tunneling diode," Phys. Rev. B 39, 7720-7735 (1989).

${ }^{10}$ P. A. Markowich, C. Ringhofer, and C. Schmeiser, Semiconductor Equations (Springer, New York, 1990).

${ }^{11}$ W. R. Frensley, “Boundary conditions for open quantum systems driven far from equilibrium," Rev. Mod. Phys. 62, 745-791 (1990).

${ }^{12}$ C. Ringhofer, D. Ferry, and N. Kluksdahl, "Absorbing boundary conditions for the simulation of quantum transport phenomena," Transp. Theory Stat. Phys. 18, 331-346 (1989).

${ }^{13}$ A. Arnold, "On absorbing boundary conditions for quantum transport equations," Math. Modell. Numer. Anal. 28, 853-872 (1994).

${ }^{14}$ P. A. Markowich and C. Ringhofer, "An analysis of the quantum Liouville equation," Z. Angew. Math. Mech. 69, 121-127 (1989).

${ }^{15}$ P. A. Markowich and P. Degond, "A quantum transport model for semiconductors: The Wigner Poisson problem on a bounded Brillouin zone," Math. Modell. Numer. Anal. 24, 697-710 (1990).

${ }^{16}$ N. Ben Abdallah, P. Degond, and P. A. Markowich, "On a one-dimensional Schrödinger-Poisson scattering model,", ZAMP 48, 135-155 (1997).

${ }^{17}$ N. Ben Abdallah, "A hybrid kinetic-quantum model for stationary electron transport in a resonant tunneling diode," J. Stat. Phys. 90, 627-662 (1998).

${ }^{18} \mathrm{H}$. Lange, B. Toomire, and P. Zweifel, "Inflow conditions in quantum transport theory," VLSI Design 9, 385-396 (1997) (special issue).

${ }^{19}$ K. M. Case and P. F. Zweifel, Linear Transport Theory (Addison-Wesley, Reading, MA, 1967).

${ }^{20} \mathrm{C}$. Bardos, "Boundary value problems for first order partial differential equations with real coefficient; approximation theorems; application to the transport equation," Ann. Scient. Ec. Norm. Sup. 4eme serie 3, 185-233 (1970).

${ }^{21}$ L. Arkeryd and A. Nouri, " $L$ solutions to the stationary Boltzmann equation in a slab," (preprint).

${ }^{22}$ R. Beals, "An abstract treatment of some forward-backward problems of transport and scattering," J. Funct. Anal. 34, $1-20$ (1979).

${ }^{23}$ W. Greenberg, C. van der Mee, and V. Protopopescu, Boundary Value Problems in Abstract Kinetic Theory (Birkhäuser, Basel, 1997).

${ }^{24}$ C. Greengard and P. A. Raviart, "A boundary-value problem for the stationary Vlasov-Poisson equations: The plane diode," Commun. Pure Appl. Math. 43, 473-507 (1990).

${ }^{25}$ C. Ringhofer, "A spectral method for the numerical simulation of quantum tunneling phenomena," SIAM (Soc. Ind. Appl. Math.) J. Numer. Anal. 27, 32-50 (1990).

${ }^{26}$ A. Arnold and C. Ringhofer, "Operator splitting methods applied to spectral discretizations of quantum transport equations,"' SIAM (Soc. Ind. Appl. Math.) J. Numer. Anal. 32, 1876-1894 (1995).

${ }^{27}$ Y. Katznelson, An Introduction to Harmonic Analysis (Dover, New York, 1976).

${ }^{28}$ C. Cercignani, R. Illner, and M. Shinbrot, “A boundary value problem for discrete-velocity models," Duke Math. J. 55, 889-900 (1987).

${ }^{29}$ S. Kawashima, “Existence and stability of stationary solutions to the discrete Boltzmann equation,” Jpn. J. Indust. Appl. Math. 8, 389-429 (1991).

${ }^{30}$ A. Pazy, Semigroups of Linear Operators and Applications to Partial Differential Equations, 2nd ed. (Springer, New York, 1992).

${ }^{31}$ M. Cessenat, "Théorèmes de trace pour des espaces de fonctions de la neutronique," C. R. Acad. Sci., Ser. I: Math. 300, 89-92 (1985).

${ }^{32}$ R. Dautray and J. L. Lions, Mathematical Analysis and Numerical Methods for Science and Technology, Evolution Problems II, Vol 6. (Springer, Berlin, 1993).

${ }^{33}$ L. Arkeryd, C. Cercignani, and R. Illner, "Measure solutions of the steady Boltzmann equation in a slab," Commun. Math. Phys. 142, 285-296 (1991).

${ }^{34}$ E. Griepentrog and R. März, Differential-Algebraic Equations and Their Numerical Treatment (Teubner, Leipzig, 1986).

${ }^{35}$ K. E. Brenan, S. L. Campbell, and L. R. Petzold, Numerical Solution of Initial-Value Problems in Differential-Algebraic Equations, Classics in Applied Mathematics Vol. 14 (SIAM, Philadelphia, 1989). 